\title{
Montelukast lindert lokale Reaktionen bei Immuntherapie
}

\section{Starke Lokalreaktionen und Juckreiz an der Einstichstelle sowie die selteneren systemischen Reaktionen sind die Hauptkomplikationen einer subkutanen spezifischen Immuntherapie. Können diese Neben- wirkungen durch eine geeignete Prämedikation gemildert werden?}

E s ist bekannt, dass Antihistaminika zur Prophylaxe von Lokalreaktionen im Rahmen einer Hyposensibilisierung zumindest teilweise geeignet sind. Da bei der Reaktion nicht nur Histamin, sondern auch Leukotriene und Prostaglandine eine Rolle spielen, erscheint es sinnvoll zu überprüfen, welche prophylaktische Wirkung ein Leukotrienantagonist entfaltet. Dieser praxisrelevanten Fragestellung haben sich jetzt Allergologen der Universität Wien angenommen.

Sie untersuchten den Effekt des Leukotrienantagonisten Montelukast im Vergleich zu Desloratadin bei Patienten, die sich wegen einer Insektengiftallergie einer Rush-Therapie unterzogen. Bei diesem Therapieverfahren kommt es relativ häufig zu Nebenreaktionen. Das Therapieschema sah 19 Injektionen bis zum Erreichen der Höchstdosis vor, die sich auf fünfTage verteilten. Bei der Auftitrierung wurden wässrige Lösungen verwendet, zur Erhaltungstherapie kamen dann Depotpräparate zum Einsatz. Die Prämedikation wurde jeweils eine Stunde vor der ersten Injektion verabreicht. Dabei gab es drei Gruppen: Die Patienten der ersten Gruppe erhielten

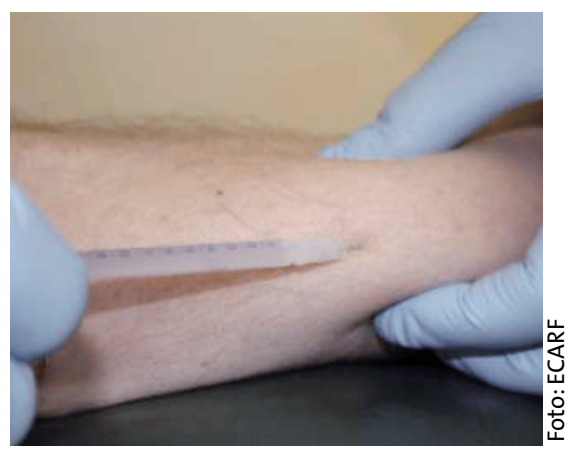

Eine Prämedikation mit Montelukast kann lokale Nebenwirkungen nach einer subkutanen Allergeninjektion abmildern.
$10 \mathrm{mg}$ Montelukast, die der zweiten $5 \mathrm{mg}$ Desloratadin und die der dritten 10 mg Laktose als Plazebo. Traten lokale Reaktionen auf, wurden diese vermessen und zugleich der Juckreiz beurteilt.

Bei der Auswertung zeigte sich, dass im Vergleich zu Plazebo in beiden Verumgruppen Lokalreaktionen erst bei höheren Allergenkonzentrationen auftraten. Montelukast war dabei statistisch signifikant effektiver als Desloratadin. Der Unterschied zwischen Desloratadin und Plazebo war dagegen nicht signifikant. Die Prämedikation mit Montelukast verringerte auch die Größe der nach sechs und nach 24 Stunden gemessenen Lokalreaktionen deutlicher als das Antihistaminikum oder Plazebo. Der Juckreiz wurde hingegen durch keine der Medikationen signifikant reduziert. Systemische Reaktionen traten unter Montelukast nicht auf. Jedoch ereignete sich in der Plazebogruppe eine mittelschwere systemische Reaktion mit Blutdruckabfall und in der Desloratadingruppe eine urtikarielle Hautreaktion außerhalb der Injektionsstelle.

\section{Fazit}

Lokalreaktion im Rahmen einer subkutanen Immuntherapie werden durch eine Prämedikation mit Montelukast effektiver verhindert als durch Desloratadin. Ein positiver Einfluss auf systemische Reaktionen scheint möglich, jedoch ist die Fallzahl dieser Pilotstudie zu klein für eine Beurteilung. Weitere Studien sollten folgen.

\section{Literatur}

Wöhrl S et al. Premedication with Montelukast reduces local reactions of allergen immunotherapy. Int Arch Allergy Immunol 2007;144: $137-42$

Dr. Dieter Bruchhausen, Wuppertal
Allergologie im Kloster 2009

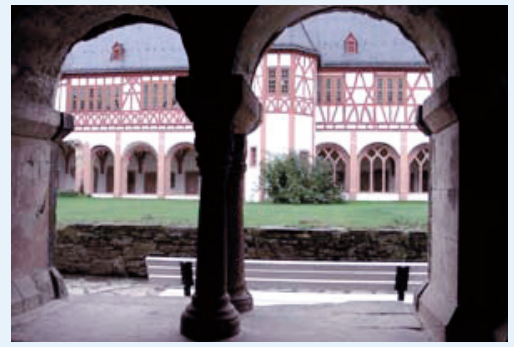

„Porta patet, cor magis“, zu deutsch „Die Tür steht offen, mehr noch das Herz!“ getreu diesem Wahlspruch der Zisterzienser wird im Kisselbachtal oberhalb von Kiedrich seit dem Jahre 1116 eine weltoffene und gastfreundliche Tradition gelebt. Seit einigen Jahren dürfen wir Allergologen jeweils im Wonnemonat Mai bei der Veranstaltung „Allergologie im Kloster" in diese Tradition eintauchen. Dieses Jahr findet das Treffen am 16. Mai statt und wieder werden im Kloster Eberbach bei Eltville im Rheingau aktuelle Forschungsergebnisse ausgetauscht werden und Weiterbildungsreferate auf hohem Niveau zu hören sein. Wichtige Themen 2009 sind: — Nahrungsmittelallergien - aktueller Stand von Diagnostik und Therapie — Sinnlose diagnostische Tests und Therapieverfahren in der Allergologie _ Therapie mit Anti-IgE-Antikörpern: Was hat sich in der Praxis bewährt? _ Update ASS-Intoleranz - Polyposis nasi und Asthma bronchiale

_ Ambrosia/Ragweed: Wie sieht das Problem wirklich aus?

_ Allergien im Kindesalter - frühzeitig erkennen und behandeln

_ Patientenmanagement in der allergologischen Facharztpraxis

Neben hochkarätigen Vorträgen erwartet Sie mit dem „Hyde Park im Klostergarten" ein Diskussionsforum der besonderen Art. Außerdem wird Ihren Arzthelferinnen mit der „Allergologie zum Anfassen“ ein sehr praxisorientiertes Intensivseminar geboten.

Im Jahr 2008 wurde erneut der Besucherrekord des Vorjahres gebrochen, auch 2009 werden wieder über 700 Allergologen aus dem gesamten deutschsprachigen Raum erwartet. Bitte melden Sie sich also frühzeitig an.

Dr. Oliver Pfaar, Prof. Dr. Ludger Klimek Zentrum für Rhinologie und Allergologie An den Quellen 10

65183 Wiesbaden

Tel.: (o 611) 8804799

Fax: (0611) 3082360

E-Mail: oliver.pfaar@hno-wiesbaden.de 